\title{
ENTRE PODER Y RESISTENCIAS: ANÁLISIS DE LAS MOVILIDADES NO TERRITORIALES EN LA ZONA FRONTERIZA DE LA REGIÓN DE TARAPACÁ, CHILE
}

\author{
BETWEEN POWER AND RESISTANCE: ANALYSIS OF THE NON-TERRITORIAL \\ MOBILITY IN THE BORDER ZONE OF THE REGION OF TARAPACÁ, CHILE
}

\author{
Romina Ramos Rodríguez*
}

\begin{abstract}
El monopolio epistemológico del territorio del Estado, en ocasiones, ha sido desarrollado como un constructo indisociable. Sin embargo, aunque el territorio es el objetivo más importante del Estado, creemos que de ninguna manera es la única forma de apropiación espacial. En consecuencia, para lograr la tarea de desmarcar este binomio como producto exclusivo, necesitamos nuevas metáforas y campos de visión que transmitan el alcance completo de una imaginación espacial no territorial. En efecto, desde una perspectiva situada, analizaremos las movilidades de los cruzadores fronterizos de la región de Tarapacá a partir del seguimiento de sus itinerarios de cruce. Con todo, conjeturamos que en el espacio fronterizo tarapaqueño cohabitan expresiones de regulación de movilidad por parte del Estado, y también de prácticas de movilidades temporales por parte de sus habitantes en una relación de permanente disputa.
\end{abstract}

Palabras claves: Territorio, frontera, movilidad, región de Tarapacá.

The epistemological monopoly of the territory of the State, at times, has been developed as an inseparable construct. However, although the territory is the most important objective of the State, we believe that it is by no means the only form of spatial appropriation. Consequently, to achieve the task of unlocking this binomial as an exclusive product, we need new metaphors and fields of vision that convey the full scope of a non-territorial spatial imagination. In fact, from a situated perspective, we will analyze the mobilities of border crossings in the Tarapacá region based on the monitoring of their crossing itineraries. However, we conjecture that expressions of mobility regulation by the State coexist in the Tarapacá border area, as well as practices of temporary mobility by its inhabitants in a relationship of permanent dispute.

Key words: Territory, mobility, border, region of Tarapacá.

\section{Introducción ${ }^{1}$}

Tradicionalmente se ha enfatizado al territorio como límite, pero no se ha realizado un análisis más fructífero que nos ayude a la compresión de las no territorialidades del Estado ${ }^{2}$.

Desde esta premisa investigativa, seguiremos la propuesta de Lefebvre (1974), quien centró su atención al espacio vivido, y discutiremos cómo a pesar de lo estructurante que puede ser la territorialización del Estado en las zonas de frontera, igualmente existen expresiones no territoriales que practican los cruzadores fronterizos, manifestadas a partir de sus frecuentes viajes a uno y otro lado del límite fronterizo, ubicado entre la región ${ }^{3}$ de Tarapacá, Chile, y la provincia de Oruro, Bolivia. Lo anterior lo desarrollamos suponiendo que habitar produce un hábitat apropiado del territorio $\mathrm{y}$, por tanto, el territorio será un fragmento del espacio apropiado y no necesariamente una totalidad (Bourdieu, 1999). Esta apropiación espacial, entendida como la materialización del poder y de la interacción entre el sujeto y campos de fuerza donde se despliega el capital ${ }^{4}$, estructura diversos imaginarios con sus consecuentes efectos sociales, políticos y culturales (Guerrero, 2016).

En este contexto de estudio, la frontera tarapaqueña la entenderemos como espacio transfronterizo cambiante ${ }^{5}$ donde surgen identidades nuevas y viejas en contacto, concebida como: "Una creación histórica, [que] aparece siempre inserta en un espacio/ tiempo en constante mutación" (Medina, 2006:14). Esta tendencia múltiple de nuestra frontera también se manifiesta en las posiciones que se desenvuelven entre los actores fronterizos en tanto relaciones sociales de poder asimétricas. Son asimétricas porque notamos que confluyen identidades nacionales, regionales, transfronterizas ${ }^{6}$, y cada una tiene su

\footnotetext{
* Universidad Arturo Prat, Facultad de Ciencias Jurídicas y Políticas. Núcleo de Estudios Criminológicos de la Frontera. Iquique, Chile. Correo electrónico: romramos@unap.cl
} 
propia representación del espacio y, como consecuencia, deviene una pugna entre una representación oficial del territorio impuesto por los respectivos Estado-naciones, y los espacios de representación (Lefebvre 1974; Filibi, 2011) construidos por una gama de actores sociales, subalternos, como es el caso de los cruzadores fronterizos que habitan/ transitan y que tienen una mirada del espacio y una identidad resultado de sus cambiantes trayectorias espaciales (Pedone, 2010).

Para analizar esta configuración, el objetivo de este trabajo es reflexionar acerca de la noción de territorio, y también de las movilidades no territoriales de los cruzadores fronterizos. Este acercamiento nos permitirá descubrir de qué forma el texto del espacio (Lefebvre, 1976), como dimensión de análisis que contiene a todos los procesos empíricos que hemos observado, adquiere una significativa posición.

La estrategia metodológica de esta investigación adhiere a las formas particulares de abordar los espacios de frontera (Marcus, 2005). En este sentido, se reconocen los análisis transfronterizos desde la etnografía multisituada, distinguiéndose de investigaciones comparativas que se concentran en los Estados nacionales en tanto unidades relativamente exclusivas y estáticas (Faist, 2012). Este tipo de investigación se interesa por explorar la circulación de objetos, mercancías, identidades y significados culturales, en marcos espacio-temporales más o menos difusos, tratándose de una etnografía móvil (Fernandiz, 2011). Para ello, las entrevistas se realizaron en la arquitectura fronteriza y en nuestro espacio fronterizo tarapaqueño, utilizando como técnica de campo la bola de nieve ${ }^{7}$. Esta técnica, también conocida como muestreo en cadena de referencia, es : "un método de recogida de investigación cualitativa de personas nuevas que han sido referenciadas por personas que ya han sido incluidas en el estudio" (López y Deslauriers, 2011: 9). Esta estrategia de caso ha sido ampliamente utilizada en el campo de las ciencias sociales, usada para acceder a personas que son parte de una "población oculta" o aquellos que a menudo no están representados en otras muestras (Blanco et al., 2007).

Con todo, el itinerario de este trabajo es el siguiente:

Primero, siguiendo el debate teórico en torno a la categoría de territorio, identificamos algunos aspectos clave acerca de la territorialización del Estado moderno en los estudios fronterizos. A continuación discutiremos la potencialidad heurística de la zona fronteriza de la región de Tarapacá. Seguidamente, interpretaremos la franja fronteriza tarapaqueña y su configuración histórica como espacio de movilidad no territorial, cotejándolo con el debate teórico antes expuesto y algunas entrevistas realizadas. Finalmente, expondremos las conclusiones del estudio.

\section{La territorialidad como ejercicio de (en) marcar: la trampa del territorio}

El primer enfoque asociado al territorio es su inminente presunción natural, influenciado en gran medida por la geografía física (Capel, 1981; Benedetti, 2011b). No ahondaremos en esta perspectiva territorial debido a que no tiene relevancia para este caso, aunque reconocemos la importancia de señalarlo como punto de partida en los estudios de geografía. El segundo, es la noción del territorio como obra del Estado y todo lo que contiene en él, en tanto soberanía ${ }^{8}$ territorial, le pertenece (Capel, 2016). En este enfoque, el poder del Estado es el que determinará quién está dentro y fuera. Esta segunda concepción territorial excluyente trató de homogenizar al territorio haciéndonos creer que aludía a un límite naturalizado (Neep, 2016). Este encuadre analítico tiene consecuencias como, por ejemplo, ignorar que el territorio es un efecto construido, en lugar de una dimensión natural del espacio, y esto, a su vez, viene con un cierto coste asociado: la asunción de la territorialidad de Estado nos puede desviar en un estancamiento intelectual que John Agnew (1994) identifica como the territorial trap.

La "trampa territorial" como magistralmente nos advierte el geógrafo John Agnew en su influyente texto acerca de los sesgos metodológicos que implica un análisis homogéneo del territorio, es la obsesiva tendencia de atribuir al Estado la exclusividad en su producción, sin considerar los imaginarios geográficos que se dibujan a partir de los sujetos que se apropian de los espacios, o los flujos de redes de distinto signo (económicas, materiales, poblacionales, etc.) que también se configuran en los territorios (Agnew, 1994).

Esta visión homogénea se basa en una ontología que hace plausibles suposiciones engañosas y generalizadas respecto del territorio. De esta forma se mencionan tres hipótesis en la que nuestras investigaciones se podrían ver envueltas en la trampa territorial de Agnew. La primera hipótesis es considerar que los Estados reclaman la soberanía de los 
territorios y su delimitación, independientemente de la gubernamentalidad (Foucault, 2002). Esta premisa da lugar a la suposición de que los territorios pueden considerarse como unidades espaciales donde los Estados chocan, en mayor o menor grado, pero en sus configuraciones no causan ningún cambio en su composición interna. La segunda hipótesis es que el Estado es visto como un actor singular que lucha con otros actores por su funcionamiento a escala geográfica y, en consecuencia, se separa en Estados "nacionales/extranjeros", oscureciendo, por ejemplo, las formas transnacionales que se han incorporado a la formación del Estado (Pedone, 2010). En tercer lugar, el Estado visto como un contendor de la sociedad, suponiendo que las fronteras territoriales del Estado representan el natural "contenedor" de las estructuras, procesos y acciones de la vida social y política de la sociedad. Este inadecuado supuesto, que hace equivalente a la sociedad y el Estado como misma unidad de análisis (Smith, 1979), nos refleja de forma involuntaria una perspectiva parcial e incompleta acerca de la naturaleza del Estado dentro de ese mundo. Tomado en conjunto, estas tres premisas conforman la "trampa territorial" (Agnew, 1994; 2015).

La territorialidad es el ejercicio de enmarcar, y el territorio es el espacio en el que se despliega la intención de ejercer un poder sobre él, de apropiarlo, dominarlo, domesticarlo (Mendiola, 2012).

Estos imaginarios se determinaron por características homogéneas, atribuibles exclusivamente a las acciones que emanan desde la estructura del Estado. Esto a su vez engendró, de un punto de vista diacrónico, que la organización de los territorios y sus engranajes político-económicos propuestos en los siglos XVIII y XIX, el desarrollo de la aspiración democrática que ostentan las sociedades actuales, incluso la producción de las identidades colectivas se expliquen, en gran medida, al nacimiento y la afirmación de los Estados nacionales (Ovando y Ramos, 2016).

Sin embargo, sostenemos que esta noción territorial no es suficiente para comprender las diversas percepciones de nuestro espacio tarapaqueño, ya que las percepciones de todos los actores no tendrían cabida en este entendimiento del espacio social, en el caso de que nos ciñéramos solo a este enfoque territorial. Por actores nos referimos, particularmente, a las elites locales que dinamizan la integración física fronteriza, a los funcionarios regionales y a los representantes del Estado, a los cruzadores de frontera que se apropian del espacio según las oportunidades que se presentan a uno y otro lado de la línea administrativa, y a los diplomáticos oficiales que desde las agencias estatales ordenan el territorio y su convivencia transfronteriza desde la capital, entre otros (Ramos y Ovando, 2016). En particular, este ordenamiento lo observamos en la región de Tarapacá cuando comprobamos que conviven contradictorios discursos que buscan: promover políticas de "frontera abierta" para una migración "ordenada y regular" y de "frontera cerrada" para la "migración ilegal", la trata de personas y el tráfico de migrantes. Un ejemplo paradigmático de lo señalado lo constituyen el Plan Frontera Norte (2011-2014) y el Plan Nacional contra el Narcotráfico (2014-2020). Concerniente a este punto, un ejemplo clave del discurso que construye el Estado en torno a la seguridad en Tarapacá es la decisión de implementar el Plan Frontera Norte en la agenda pública de esta región (Ovando y Ramos 2016). En octubre de 2011, el primer gobierno de Sebastián Piñera anunció la orientación del Plan hacia el control de amenazas no convencionales provenientes del crimen organizado, el tráfico de drogas y las migraciones "ilegales" presentes en la región de Tarapacá (García Pizón, 2016). Este Plan pretendió asegurar mayores rangos de control de acceso a fronteras terrestres, sobre la base de implementación de tecnología, más el concurso de diversas agencias estatales que se coordinan para compartir información. Su finalidad era crear, en el terreno mismo, las condiciones necesarias para sellar los límites, anticipándose así a las fuentes de riesgo o, en su defecto, mitigarlos, disminuirlos o contenerlos (Leyton, 2011). De este modo, Tarapacá y sus condiciones hacen posible que opere la lógica gubernamental del Estado desde una expresa materialidad que hace funcionar a la frontera como un dispositivo de control (Deleuze, 2006) y, que a su vez, opera en función de proyectos dictaminados desde los gobiernos centrales hacia las zonas periféricas.

Sin embargo, nuestra concepción de espacio fronterizo es que los discursos nacionales heredados no constituyen necesariamente un imaginario nacional contenido y producido en nuestro espacio local. A diferencia de la pretensión de dominar el espacio que persigue la territorialización del Estado, el espacio apropiado presupone una heterogeneidad inherente y, por ello, en su interior contiene "diferentes espacios que se interpenetran entre sí y se superponen entre ellos" (Lefebvre, 1979:86). 


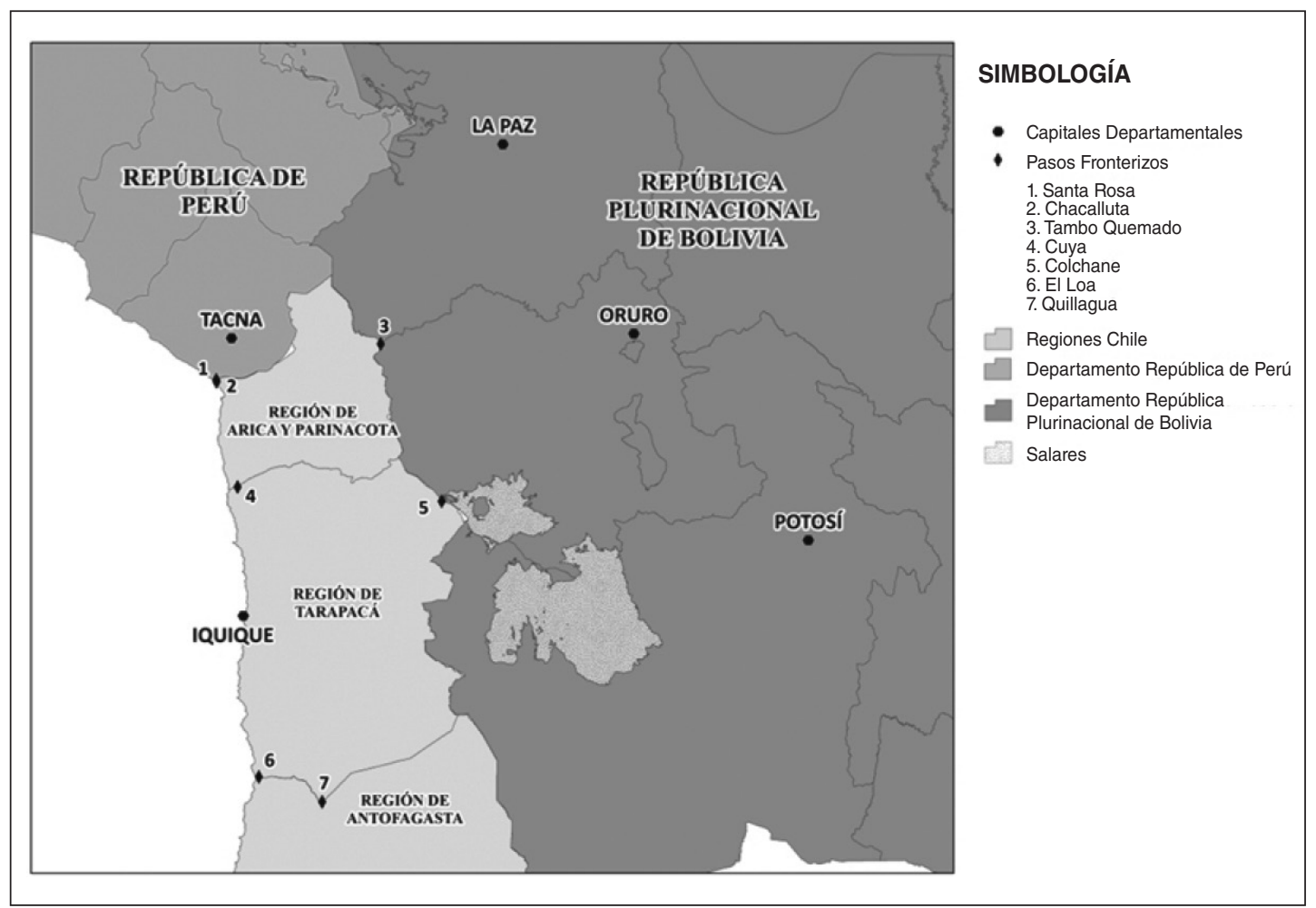

Figura 1. Mapa de la franja fronteriza de la región de Tarapacá.

Fuente: Marcela Tapia Ladino y Romina Ramos Rodríguez, "Mujeres migrantes fronterizas en Tarapacá a principios del siglo XXI. El cruce de las fronteras y las redes de apoyo", Polis [En línea], № 35, 2013.

Ahora bien, volviendo a la trampa territorial, ¿Cómo la evitamos? Daniel Neep (2016) nos propone evitarla por medio de tres análisis. El primero es la producción del espacio y la crítica a la noción de territorio del Estado moderno como un fetiche espacial al servicio de este. El segundo intento es que la noción del fetiche ayuda a analizar la formación del Estado como un proceso espacial y no exclusivamente territorial. El tercer paso es intentar explicar el espacio del Estado desde sus no territoriales, identificando casos empíricos de modalidades no territoriales del poder del Estado. En este punto, es que en el intento de no caer en fetichismos territoriales (Benedetti, 2011b; Daniel Neep 2016), hemos elaborado el acercamiento de nuestro estudio hacia la comprensión del migrante transfronterizo, que en su movimiento constituye no territorialidades mediante sus itinerarios de cruce.

Para explorar este funcionamiento no territorial del espacio del Estado, debemos centrarnos menos en la extensión nacional de las instituciones del Estado y, en su lugar, asistir a las irregulares y variadas alteraciones espaciales (Neep, 2016). O también, como nuestra opción investigativa, ahondar en nuestras grietas fronterizas (Grimson, 2000a; Mignolo, 2003). Reemplazar la lente territorial del Estado-Nación por una que sea más inclusiva espacialmente, abrirá la "caja negra" del Estado y permitirá una construcción descentralizada y heterogénea de múltiples salidas.

\section{Potencialidad heurística del espacio fronterizo tarapaqueño}

La región de Tarapacá es hoy una de las quince regiones que forma parte de la división políticoadministrativa de Chile. Desde el 2007 se escinde territorial y administrativamente de la Región de Arica y Parinacota. A continuación mostramos la región y sus administraciones territoriales (Figura 1).

Como podemos ver en la figura anterior, nuestro espacio de interés -Tarapacá- limita al norte con la Región de Arica y Parinacota, al sur con la Región de Antofagasta, al este con el Estado 
Plurinacional de Bolivia y al oeste con el océano Pacífico. Cuenta con una superficie de $42.225,8 \mathrm{~km}^{2}$ que representa el $5,57 \%$ del territorio nacional. La región está compuesta por las provincias de El Tamarugal e Iquique y la capital regional es la ciudad de Iquique. Su población estimada es de 330.558 habitantes (INE, 2018). En tanto su población extranjera representa el 14\%, superando ostensiblemente la media nacional que representa 4\% (INE, 2018). En la revisión de los datos del Censo 2017 se aprecia que del total de extranjeros censados en la región (43.646 personas), 75\% son de origen fronterizo, es decir, 32.752 personas, de estas, el 44\% corresponde a bolivianos, el 30,6\% es peruano (INE, 2018). Esta situación responde, en parte, al proceso histórico tarapaqueño que ha configurado y configura las tendencias espaciales de movilidad humana presentes en la zona: "sometida a los influjos propios del fenómeno de la globalización económica y al crecimiento demográfico" (Díaz y Sánchez, 2016:3).

A partir de la década de 1970 emerge la apertura económica como determinante para la conformación espacial y los imaginarios geográficos posibles de Tarapacá. La regionalización territorial, bajo un criterio neoliberal (Amhilat Zsary, 1997; Podestá, 2004), fue sometida a un proceso que promovió la inversión extranjera mediante varias medidas como fue decretar la Zona Franca en 1975 (Amhilat Szary, 1997, González, et al., 2008). Una de las principales consecuencias fue un impacto clave en su dinámica territorial en la medida que facilitó la movilidad de capitales (a partir de las reexpediciones de mercancías de la Zona Franca a Bolivia, Paraguay y Brasil), pero limitó la movilidad humana de sectores no conectados a las redes comerciales (Ovando, 2012). Así, constatamos que las nuevas reglas económicas no buscaron promover el beneficio de las poblaciones de frontera, sino que por el contrario, buscó promover el comercio por tierra entre los países que están atravesados por estas ciudades, concibiéndolas únicamente como zonas de servicios, facilitando la circulación de mercancía de las grandes empresas transnacionales (Ovando y Ramos, 2016).

A pesar de estos factores, durante la última década la región de Tarapacá se ha convertido en un destino privilegiado para los migrantes transfronterizos (Grimson y Lube, 2015). Se trata de los migrantes laborales del Cono Sur, que trabajan en países del hemisferio norte $y$, en menor escala, están optando por trasladarse a países como Chile por la particularidad señalada de las regiones como Tarapacá (Tapia y Gavilán, 2006). Esta situación responde, en alguna medida, a que la migración de América Latina se ha visto diversificada sobre todo en años recientes. Por un lado, observamos cómo el contexto de restricciones y crisis económicas de los países del hemisferio norte influye en las decisiones al momento de emigrar (Tapia, 2012; Tapia y Ramos, 2013) y, por otro lado, vemos cómo esta diversificación se materializa debido a los procesos de integración económica de la región latinoamericana que han llevado a cabo la mayoría de los países en las últimas décadas (CAN; CLARICOM; ALBA; MERCOSUR; GELAC; etc.), y que han permitido -alguno con mayor éxito que otro- que la movilidad de bienes y personas sea más dinámica debido a la existencia de una historia compartida de relaciones económicas y sociales, entre otros factores (Nicolao, 2011).

En suma, para explorar el funcionamiento no territorial en nuestro espacio fronterizo debemos centrarnos menos en la extensión nacional de las instituciones del Estado y, en su lugar, asistir a las irregulares y variadas alteraciones espaciales (Neep, 2016). O también, como nuestra opción investigativa, ahondar en nuestras grietas fronterizas (Mignolo, 2002a). Reemplazar la lente territorial del Estado-Nación por una que sea más inclusiva espacialmente, abrirá la "caja negra" del Estado y permitirá una construcción descentralizada y heterogénea de múltiples salidas.

En consecuencia, a partir de esta distinción conceptual es como adherimos a "una epistemología para la geografía" (Zusman, 2013:53) que tome en cuenta la subjetividad, y sus prácticas, como elemento clave en la diferenciación espacial. Una diferenciación que tiene que ver con las formas de imaginar el medio, de aproximarse y actuar en él, en la medida en que "la imaginación es una facultad a partir de la cual se puede otorgar sentido a las acciones, a los pensamientos y a las materialidades pretéritas, siempre permeadas por las visiones del presente" (Ibídem, 2013:54). De este modo, ponemos en cuestión la territorialidad del Estado, socavada por el nacionalismo que subyace los trabajos de las ciencias sociales en la era de "lo nacional"9 (Tapia, 2012).

Desde esta perspectiva crítica, el territorio no sería ontológicamente anterior a la acción social; por el contrario, el territorio es históricamente 
contingente y lo crean las estrategias, proyectos y tecnologías que moldean nuestra percepción acerca de él (Capel, 1981). Lefebvre (1976) señala que el espacio es producido, en lugar de pre-dado, e ilustra la tendencia recurrente de las categorías analíticas de la teoría del Estado, agregando que las tecnologías del Estado pueden ser dirigidas a producir un espacio abstracto homogéneo del territorio, aunque esto no implique que el territorio es la única forma de espacialidad del Estado.

\section{Las movilidades transfronterizas en Tarapacá y sus expresiones no territoriales}

En la actualidad el movimiento de personas por las fronteras políticas ha cambiado, acarreando varios fenómenos sociales y culturales, como por ejemplo, la nueva forma de entender los circuitos migratorios de los migrantes en tránsito (Tapia y Ramos, 2013). Es decir, personas que en su movimiento superan las fronteras nacionales y van construyendo espacialidad a partir de las prácticas culturales que mantienen y que no se pierden en los itinerarios del viaje.

En definitiva, hoy la clave estaría en presenciar que la movilización de capitales es fluida alrededor del globo para poder maximizar su rentabilidad, como también en presenciar que la fuerza del trabajo contenía y contiene grandes trabas que minimizan su valor. La movilidad de las personas y las cosas a partir del capitalismo, y como consecuencia la formación de los mercados regionales (Unión Europea, Mercosur, Asia Pacífico, etc.), se ha visto amputada, pues separa las materias primas que se trabajan en los medios productivos de la misma fuerza del trabajo que las origina (Stefoni, 2014).

Ante estos evidentes desequilibrios y tensiones es posible preguntar: ¿cómo hacemos frente a estas contradicciones de la movilidad humana? Quizás una respuesta pertinente sea el análisis de las movilidades a partir de una antropología también móvil que permita cuestionar la ilusión de un mundo sedentario (Tarrius, 2001). Los estudios antropológicos del movimiento se han centrado en las sociedades receptoras. Sin embargo, a nosotros nos interesa el movimiento que se constituye en las experiencias en el tránsito hacia el destino. Considerar que las personas se mueven es considerar también que la cultura se mueve, porque las personas trasladan valores, tradiciones y rituales en su movilidad que, a su vez, la reproducen en otros espacios; aunque su intensidad varíe, el acarreo cultural no desaparece (Tapia y Ramos, 2013).

Dentro de esta movilidad internacional existe una literatura tradicional que suele analizar, sobre la base de dos grandes categorías, los distintos tipos de migración. Por una parte, la "migración permanente" que comprende a quienes cambian de manera definitiva su residencia, región o país habitual (Stefoni, 2010). Por la otra, encontramos la comprensión de las movilidades desde la "migración temporal" o "circular" (Contreras, et al., 2017), referida a aquellos desplazamientos continuos y recurrentes que definen un constante ir y venir, pero manteniendo la residencia habitual en la comunidad de origen: "Se trata de cambios de residencia que no alteran el carácter permanente de la residencia habitual" (Canales y Zlolniski, 2001: 223, Tapia y Ramos, 2013). Esto último tiene un reflejo evidente en nuestro campo de investigación.
"Actualmente yo salí de viaje, viernes no más que pasó (sic), pedí permiso a mi jefe porque tengo que salir urgente y no podré trabajar, me descuenta o le doy otro día mi trabajo, como los sábados trabajo tres horas no más, tengo que subir [a la frontera chileno-boliviana] urgente el viernes, a la 1 o 2 de la mañana de viaje porque tengo que sacar a mi hija, le dieron una visa de tres meses no más"10.

Hemos detectado mediante los relatos de las personas entrevistadas que esta situación de temporalidad se refleja en la movilidad fronteriza tarapaqueña, donde la persona trabaja de lunes a viernes a un lado de la frontera y regresa a su hogar durante el fin de semana. Lo anterior, lo observamos en el siguiente relato:

"Mi meta de mi (sic) era sacar papeles y viajar a ver a mis hijos y volver a trabajar y volver a ver a mis hijos y así.... Entonces estoy esperando que salga un trabajo en que paguen bien con $\$ 350.000$ me conformo y tiempo completo, mientras voy y vuelvo seguido, digamos todas las semanas"11.

De acuerdo con nuestras entrevistas, es reconocida esta práctica de habitar el espacio de forma temporal ${ }^{12}$; los medios de transporte, la 
pavimentación de caminos, los medios de comunicación, en conjunto con las posibilidades económicas de uno y otro lado del límite fronterizo, permiten activar estas estrategias de cruce. Esta situación la podemos ver reflejada en los siguientes relatos que hemos rescatado de las entrevistas a los oficiales que trabajan en el complejo fronterizo de Colchane y a una mujer de nacionalidad boliviana que cruza la frontera de manera habitual:

"Hay gente cumple sus 90 días y salen y vuelve a ingresar, para trabajar por periodos cortos pero en definitiva cumplen los 90 días" 13 .

"Yo y mis hermanos siempre hemos venido a Chile, de pequeñitos, con mi mamá y luego ya solitos. Somos 8 hermanos, no había dinero entonces alguien nos dijo de venir a trabajar. Yo ahora vengo dos meses y me voy quince días para que no tenga problemas con mi visado y aprovecho de estar con mis hijos y ver la construcción de mi casa nueva que estamos haciendo"14.

De esta forma, si pretendemos analizar la frontera y a su vez la movilidad que en este espacio se manifiesta, ambas expresiones resultan ser indisociables porque no podemos referirnos a la frontera sin la noción de movilidad: "Las fronteras pueden pensarse como dispositivos creados por los Estados, localizados sobre los límites internacionales para inmovilizar todo lo que ingresa/egresa desde el interior del territorio desde/hacia el vecino" (Benedetti, 2014:37). Es decir, la frontera en sí misma es utilizada tanto para movilizar capitales, como para inmovilizar personas, creando este dispositivo en función del interés del que lo maneja: "El fin último de la gestión de la movilidad humana es filtrar selectivamente a los migrantes en términos productivos. No se trata, por tanto, del bloqueo de la movilidad, sino de su manejo" (Balibar, 2003:108). El poder del Estado es que tiene la capacidad de permitir qué bienes y personas se muevan o no por estos espacios. El manejo en las restricciones de la circulación de personas se evidencia en los siguientes relatos que hemos rescatado en nuestras entrevistas:

"Me presenté dos veces en Chacayuta y me rechazaron, nunca nos dijeron el por qué, simplemente no pasen y no pasas y ya, te rechazan...cuando me presenté por Bolivia, que de hecho intenté hacerlo otra vez por la vía legal... y no ingresé de la mejor manera, simplemente tratan a uno de la peor manera y no te dan explicaciones, no te dan opción de nada simplemente eres rechazado y ya...y en Colchane había una cantidad de gente impresionante" 15 .

Henri Lefebvre nos advertía que: "los espacios fronterizos son lugares de confluencia de flujos, enlazamiento de redes y de intercambios sociales que van más allá de la obvia dinámica comercial" (1974:220). A partir de esto, podemos suponer que no es casual que los gobiernos de las dictaduras latinoamericanas dedicaran una inusitada importancia a las fronteras, pues entendían que poseían una capacidad de ser espacios susceptibles de expresiones heterogéneas en respuesta a la intención del imaginario homogéneo de los Estados nacionales (Benedetti, 2011a). En suma, a pesar de que la percepción de las fronteras en América del Sur y Chile ha variado respecto de su utilización, continúa imaginándose como un territorio peligroso que se debe cautelar mediante el control del flujo y movimiento. Lo anterior lo vemos reflejado en el siguiente apartado que hemos rescatado de un funcionario que trabajó en zonas de frontera:
"Depende de la ocasión, pero sí que es cierto que nosotros creemos que las fron- teras son peligrosas porque aquí en Chile no es como en otro países. Por ejemplo, yo le pregunto a Ud., cuántos países tiene 5 mil km de frontera con otros países? Entonces, es obvio que nuestras zonas de frontera constituyen un peligro para Chile. $\mathrm{O}$ sea, es un riesgo y una oportunidad. Oportunidad porque tenemos un país largo y eso nos facilita de recursos naturales, por ejemplo. Pero también es un peligro porque no podemos controlar todo el territorio, eso es imposible, muy caro, se necesitará mucho personal. Entonces qué pasa, pasa que ellos, los traficantes de lo que se le ocurra (sic) lo saben muy bien" 16 .

De acuerdo con los relatos anteriormente evidenciados, comprendemos que nuestro espacio fronterizo es apropiado por determinadas relaciones sociales que lo producen $\mathrm{y}$, a su vez, lo mantienen 
desde formas de poder: "El territorio es, al mismo tiempo, una convención y una confrontación, porque el territorio posee límites, posee fronteras, es un espacio de conflictos" (Mancano, 2005:3. Así, el territorio es un espacio de libertad y dominación, de expropiación y resistencia, así como señala Foucault (2002): donde hay relaciones de poder hay resistencias al poder.

Siguiendo esta premisa foucaultiana, y proyectándola en el ejemplo del espacio social tarapaqueño, en las sociedades que se encuentran altamente influenciadas por el modelo económico neoliberal (como la nuestra), el espacio también tiene el poder de dotar de un estatus socioeconómico a quien lo habita. El espacio, igualmente, se practica acorde a los distintos gradientes de movilidad otorgados.

Esta restricción en el movimiento es una de las intenciones en la creación de generar fronteras políticas. Sin embargo, debido a las características propias de la zona fronteriza tarapaqueña que ya hemos señalado, vemos en los relatos anteriores que la migración transfronteriza erosiona el imaginario de movilidad permanente con el objetivo último de permanencia definitiva y, además, erosiona la intención del dispositivo de control fronterizo, en tanto que las restricciones no bloquean la movilidad, sino que estos mecanismos de control propician la elaboración de otras estrategias de cruce que rebasan ese imaginario securitario del Estado (Bigo, 2008).

Entonces es factible preguntar ¿cómo se vincula movilidad transfronteriza con la construcción de la espacialidad en la franja fronteriza de Tarapacá? La respuesta la encontramos desde el "paradigma de la movilidad" de Tarrius (2000), donde se aprecia a la movilidad como aquellos movimientos de personas "móviles" que generan territorios, en contraposición al "paradigma del lugar" que alude fundamentalmente a lo sedentario o el establecimiento.

Se trata de varias movilidades que no tienen como fin la instalación o un proyecto migratorio que implique establecimiento, pero que, sin embargo, desde el paradigma de la movilidad generan e instauran territorio (Tapia, 2012, 2017). Tarrius denomina este enfoque como una "antropología del movimiento", proponiendo a las movilidades humanas como "... productoras y estructurantes de formas de sociabilidad no localizadas y que, por tanto, dan lugar a territorios circulatorios que surgen como efecto y condición de prácticas de movilidad" (2000:48). La movilidad en las franjas fronterizas se concibe como una categoría de análisis que advierte que no es que se construya cotidianeidad fuertemente arraigada en lo establecido, más bien se producen y configuran apropiaciones espaciales, en la medida en que las personas transitan y con ese tránsito van constituyendo una realidad histórica cambiante.

De este modo, los lugares de frontera se vuelven atractivos por diferentes razones; algunas derivadas estrictamente de su posición en el borde del territorio (camiones que van hacia allí simplemente para cruzar hacia el otro país), otras, por la multiplicidad de relaciones espaciales que establecen los lugares fronterizos y que son generadoras de una multiplicidad de trayectorias que, mediante actividades comerciales informales, reflejan una vida cotidiana marcada por la inexistencia de fronteras políticas:

"Yo le decía señorita, que yo siempre he viajado y cruzado, incluso antes cuando no estaba ese edificio (complejo fronterizo) y solo había como un control pequeñito, pero claro, yo viajaba a trabajar por la temporada de verano y luego volvía por mis papás que estaban enfermitos, entonces yo siempre he viajado para aquí y regreso a mi país"17.

Consecuencia de todo lo anteriormente expuesto es que nos hemos centrado en conocer la subjetividad de sentido que los actores le atribuyen a sus trayectorias en tanto movilidad fronteriza, lo que nos permite establecer que, "los viajes no se distinguen ni por la cualidad objetiva de los lugares ni por la cantidad mensurable del movimiento (...) sino por el modo de espacialización, por la manera de estar en el espacio, de relacionarse con el espacio" (Deleuze y Guattari 1988:490).

En suma, creemos que la movilidad concebida como una manifestación empírica de apropiación de espacio involucra, por un lado, la pretensión de pautar la movilidad por parte del Estado que, por medio de su noción de territorio, establece regímenes de control y, por otro lado, como reflejo encontramos la resistencia espacial observada en las no territorialidades de los cruzadores fronterizos que se apropian del espacio en tanto transitan.

Esta dialéctica pone en juego las necesidades de los sujetos para desarrollar sus actividades de 
reproducción de la vida social en un contexto territorial y temporal. En consecuencia, es necesario pensar en la capacidad de los migrantes fronterizos para construir, a partir de sus itinerarios de cruce, nuevos territorios como un potencial que emerge desde las grietas de la movilidad, "que tensionan las certezas y redefinen con ello los contornos y los fundamentos del Estado moderno (...). Se trata de prácticas de control y resistencia que entran en juego en los actuales movimientos migratorios" (Stefoni y Stang, 2017:119).

Analizar las no territorialidades (movimientos, flujos, redes, cruces fronterizos breves, etc.) considerados como espacios de resistencia, nos permite reorientar la investigación empírica acerca de la noción del territorio del Estado. Hasta hace poco tiempo, estas apropiaciones del espacio se consideraban marginales y, por tanto, de un menor interés investigativo. En efecto, estas expresiones materiales y simbólicas del espacio fronterizo irrumpen en los imaginarios geográficos y adquieren un sentido singular en la conformación del imaginario nacional respecto de la noción de territorio. Todas estas expresiones de resistencia que se dibujan en los espacios como el nuestro, que muchas veces son generadoras de conflicto, como hemos podido apreciar por medio del relato de nuestros entrevistados, responden en mayor o menor grado a que los Estados en estos territorios marginales no se han retirado, sino que han sustituido un modelo de territorialización por otro; el control de las fronteras por su inminente amenaza a la seguridad nacional en un contexto de las dictaduras militares, para pasar a una territorialización donde el Estado se interesa en el control “obsesivo" de los flujos (Grimson, 2004).

\section{Conclusiones}

Hemos visto que la territorialización del Estado moderno impuso una comprensión acerca del territorio y homogenizó a la sociedad. Esto provocó un fetichismo estatal y que los estudios en ciencias sociales dialogaran en esta clave ideológica. Este fetichismo asumió que los contornos sociales coincidían con los del Estado-Nación y desconoció el carácter heterogéneo de los espacios.

Para revertir esta noción del espacio fronterizo $\mathrm{y}$ alejarnos de la trampa territorial que nos advierte Agnew (1994, 2005), hemos querido avanzar en el estudio de frontera desde sus propias particularidades $\mathrm{y}$, sin desconocer la influencia de las posiciones centrales, mostrar otra forma de entender nuestros territorios.

En este sentido, de acuerdo con la influencia de la memoria territorial, hemos descubierto que el factor temporal de las movilidades espaciales es un acercamiento interesante en términos teóricos que debemos considerar al momento de emprender estudios fronterizos. Lo anterior cobra sentido si corroboramos que la cercanía facilita la movilidad en la frontera de Tarapacá, cambiando el imaginario tradicional de los estudios migratorios que develaban que el proyecto de movilidad era de un arraigo por largo tiempo. Vemos, por los relatos, que el establecimiento definitivo no siempre es una opción. Apreciamos, en esta misma línea, que las movilidades temporales también son una expresión migratoria que carga de sentidos y significados a quien los utiliza. Estas prácticas se relacionan con la posibilidad de encontrar mejores condiciones de vida en otros espacios, superando los límites fronterizos, pero que en sus movimientos también trasladan valores y sentidos e igualmente instauran territorio a partir de estos breves itinerarios de cruce. De esta forma, los habitantes, conscientes del abandono de sus respectivos Estados nacionales, cruzan la frontera y construyen su vida cotidiana en tanto oportunidades que se manifiestan a uno y otro lado del límite administrativo. Esta situación supone una concepción común del espacio a la hora de materializar el cruce.

Con todo lo anteriormente expuesto, consideramos que la territorialización no ha desaparecido, más bien, ha cambiado. Esta premisa investigativa nos acerca al análisis de las movilidades fronterizas desde una perspectiva múltiple, donde apreciamos que el migrante fronterizo es portador de espacio y su movimiento constituye un caso empírico de no territorialidad. Este análisis lo desarrollamos cuestionando aquella premisa que sostiene que solo el Estado produce territorio, desviándonos de la trampa territorial que pretendemos superar. Nuestra intención ha sido no caer en la interpretación fetiche del Estado moderno, pero tampoco suponer falsos romanticismos. Ya que, como hemos visibilizado mediante los discursos, deben generar estrategias de resistencia para superar las restricciones impuestas en este dispositivo llamado frontera. De esta forma, despojamos a la movilidad de idealizaciones soñadoras propias del viejo imaginario moderno (Mendiola, 2012), para pasar a atender en lo concreto a la movilidad como una 
práctica situada en y sobre el espacio. A partir de esta premisa, corroboramos que surgen territorios originales que dibujan contornos móviles, como es el caso de las sociedades de migrantes en permanente movimiento, donde la mayor o menor intensidad de las movilidades fronterizas, la construcción de infraestructura de integración física, el desarrollo económico circunvecino y la relevancia geopolítica, han provocado un mayor o menor crecimiento de estos lugares, contribuyendo, por tanto, a la configuración de los espacios fronterizos como realidades heterogéneas.

\section{Referencias Citadas}

Agnew, J.

1994 "The territorial trap: the geographical assumptions of international relations theory". Review of international political economy, 1 ( $\left.\mathrm{N}^{\circ} 1\right)$, pp. 53-80.

Agnew, J.

2005 Geopolítica: una re-visión de la política mundial. Trama editorial, Madrid.

Agnew, J.

2006 "Entre la geografía y las relaciones internacionales". Tabula rasa $\left(\mathrm{N}^{\circ}\right.$ 5), pp. 85-98.

Agnew, J.

2015 "Revisiting the territorial tramp". Nordia Geographical Publications 44 ( $\left.\mathrm{N}^{\circ} 4\right)$, pp. 43-48.

Balibar, É.

2003 Nosotros, ¿ciudadanos de Europa? Editorial Tecnos, Madrid.

Blanco, M. C. M. C., \& Castro, A. B. S.

2007 El muestreo en la investigación cualitativa, pp. 27.

Benedetti, A.

2011b Territorio, lugar y paisaje. Prácticas y conceptos básicos en geografía. Editorial Facultad de Filosofía y Letras. Universidad de Buenos Aires, Buenos Aires.

Benedetti, A

2014 "Espacios fronterizos del sur sudamericano: Propuesta de un modelo conceptual para su estudio". Estudios fronterizos, 15 ( $\left.\mathrm{N}^{\circ} 29\right)$, pp. 11-47.

Contreras, Y.; Tapia, M. y N. Liberona

2017 "Movilidades y prácticas socioespaciales fronterizas entre Arica y Tacna. Del sentido frontera a la transfrontericidad entre ciudades". Diálogo Andino (Nº 54), pp. 127-141.

Bigo, D.

2008 "Globalized (in) Security: the Field and the Ban-opticon". Review Terror, insecurity and liberty: illiberal practices of liberal regimes after.9 $\left(\mathrm{N}^{\circ} 11\right)$, pp. 10-48.

Bourdieu, P.

1999 Efectos de lugar. en Bourdieu, La miseria del mundo, Fondo de Cultura Económica, Buenos Aires.

Canales, A. y Zlolniski, C.

2001 Comunidades Transnacionales y Migración en la Era de la Globalización. En: Notas de población ( No 73), CEPAL.

Capel, $\mathrm{H}$.

1981 Filosofía y ciencia en la geografía contemporánea: una introducción a la geografía, Editorial Barcanova, Barcelona.

Capel, $\mathrm{H}$.

2016 "Las ciencias sociales y el estudio del territorio". Revista bibliográfica de geografía y ciencias sociales, XXI $\left(\mathrm{N}^{\circ} 1\right)$, pp. 149. Universidad de Barcelona.

Deleuze, G., y Guattari, P. 1988 Mil mesetas. Pre-Textos.
Deleuze, G.

2006 "Post-scriptum sobre las sociedades de control". Polis, revista latinoamericana de ciencias sociales $\left(\mathrm{N}^{\circ} 13\right)$, pp. 6 .

Díaz, A. y Sánchez, E.

2016 (Editorial) "migración, fronteras, asentamientos e indígenas". Revista Diálogo Andino (N 51), pp. 3-4.

Faist, T.

2012 "Toward a Transnational Methodology: Methods to Address Methodological Nationalism, Essentialism, and Positionality". Revue européenne dems migrations internationales, Vol. 28 (1), pp. 51-70.

Fernadiz, F.

2011 Etnografías contemporáneas. Anclajes, métodos y claves para el futuro. Barcelona. Ed Anthropos Editorial. MéxicoIzalapa. División de Ciencias Sociales y Humanidades, 2011.

Filibi, I.

2011 "Innovación y desarrollos democráticos en la cooperación transfronteriza. Pueblos y fronteras en los Pirineos y el altiplano andino". Madrid: Fundamentos, pp. 117-129.

Foucault, M.

2002 La hermenéutica del sujeto. Fondo de la Cultura Económica, México.

García Pizón, V.

2016 "Territorios fronterizos: Agenda de seguridad y narcotráfico en Chile: El Plan Frontera Norte". Estudios internacionales (Santiago), 47(181), pp. 69-93.

Grimson

2000a "Pensar las fronteras desde las fronteras". Nueva Sociedad ( $\left.\mathrm{N}^{\mathrm{o}} 170\right)$, pp. 162-167.

Grimson

2000b "La fabricación cotidiana de la frontera política". Ponencia presentada en el encuentro de la Latin American Studies Association, marzo 16 y 18 de 2000, Miami.

Grimson

2004 "Fronteras, Nación y Región”. Agenda Posneoliberal. Clacso. Ponencia presentada en el Foro Social de las Américas pp. 28.

Grimson, A. y Lube, M.

2015 "Introducción. Matices y límites del transnacionalismo: los contextos de la migración en Chile". En Las fronteras del transnacionalismo. Limites y desbordes de la experiencia migrante en el centro y norte de Chile, editado por Menara Lube Guizardi, 13-34. Santiago: Universidad de Tarapacá / Ocho Libros.

Instituto Nacional de Estadísticas

2018 Resultados del Censo de población 2017. En línea: https://resultados.censo2017.cl/Region?R=R01 (visitado el 10/05/2018). 
González, M., Rouviere y Ovando, C.

2008 'De 'Aymaras en la frontera' a 'Aymaras sin fronteras'. Los gobiernos locales de la triple-frontera andina (Perú, Bolivia y Chile) y la globalización". Diálogo Andino No 31 . Guerrero, V.

2016 "Las recientes transformaciones en Iquique: el nuevo sujeto costero. Norte de Chile". Diálogo Andino ( No 51), pp. 73-80.

Lefebvre, $\mathrm{H}$.

1974 La production de l'espace, Anthropos, Paris (1976b). "Espacio y política" (traducción González Pueyo). Historia, ciencia y sociedad, 128, Ediciones Península, Barcelona.

Lefebvre, $\mathrm{H}$.

1979 Space: social product and use value. En J.W. Freiburg (ed.) Critical Sociology: European perspectives. Nueva York: Irvington, pp. 285-295.

Leyton, C.

2011 Chile y Perú: una rivalidad duradera. Ediciones Akhilleus, Santiago de Chile.

López, R. y Deslauriers, J.

2011 "La entrevista cualitativa como técnica para la investigación en Trabajo Social". Revista Margen de Trabajo Social y Ciencias Sociales, pp. 2-19.

Mancano, B.

2005 "Movimientos Socio-territoriales y Movimientos Socio-espaciales. Contribución teórica para una lectura geográfica de los movimientos sociales". OSAL año VI ( $\left.{ }^{\circ} 16\right)$, pp. 273-283.

Marcus, G.

2005 "Ethnography in/of the World System: The Emergence of Multi-Sited Ethnography", Annual Review of Anthropology, vol. 24, pp. 95-117.

Medina, E.

2006 "Aportaciones para una epistemología de los estudios sobre fronteras internacionales". Estudios Fronterizos. 7( $\left.\mathrm{N}^{\circ} 13\right)$, pp. 9-27.

Mendiola, I.

2012 "Regímenes de movilidad y domesticación del espacio". Política y Sociedad, pp. 433-453.

Mignolo, W.

2002a "The Geopolitics of Knowledge and the Colonial Neep, D. Difference". South Atlantic Quarterly. 101( $\left.{ }^{\circ} 1\right)$, pp. 57-96.

2016 "State-Space beyond Territory: Wormholes, Gravitational Fields, and Entanglement". Journal of Historical Sociology.

Nicolao, J.

2011 "Migraciones intrarregionales en Sudamérica". Boletín Elcano (No 134), pp. 10.

Ovando, C.

2012 "La seguridad internacional en la proyección de Chile hacia el cono sur: ¿desde la doctrina de la seguridad nacional hacia la construcción de comunidades de seguridad o la emergencia de la securitización?". Revista de Relaciones Internacionales, Estrategia y Seguridad, 7(№ 2), pp. 193-219.

Paasi, A.

2009 The resurgence of the 'Region' and 'Regional Identity': theoretical perspectives and empirical observations on regional dynamics in Europe Volume 35, Issue S1, February 2009, pp. 121-146.

Pedone, $\mathrm{C}$.

2010 "Cadenas y redes migratorias: propuesta metodológica para el análisis diacrónico-temporal de los procesos migratorios". Empiria. Revista de metodología de ciencias sociales $\left(\mathrm{N}^{\circ} 19\right)$, pp. 101-132.

Smith, A.

1979 Nationalism in the Twentieth Century. Oxford.

Stefoni, C.

2004a "Inmigrantes transnacionales: la formación de comunidades y la transformación en ciudadanos". Facultad Latinoamericana de Ciencias Sociales, sede Chile [en línea]. Fuente: http://bibliotecavirtual.clacso.org.ar/ar/libros/chile/ flacso/artstef.pdf (consultado en octubre de 2017).

Stefoni, C.

2014 "Perspectiva transnacional en los estudios migratorios: Revisión del concepto y nuevos alcances para la investigación”. Poblaciones en movimiento: Etnificación de la ciudad, redes e integración. Ediciones Universidad Alberto Hurtado. Santiago de Chile, pp. 41-65.

Stefoni, C. y Stang, F.

2017 "La construcción del campo de estudio de las migraciones en Chile: notas de un ejercicio reflexivo y autocrítico". ÍconosRevista de Ciencias Sociales [S.1.], (N 58), pp. 109-129.

Tapia, M. y Ramos, R.

2013 "Mujeres migrantes fronterizas en Tarapacá a principios del siglo XXI: El cruce de las fronteras y las redes de apoyo". Polis (Santiago), 12(No 35), pp. 229-257.

Tapia, M.

2012a "Frontera y migración en el Norte de Chile a partir del análisis de los censos de población. S. XIX y XXI". Revista Geografía Norte Grande (No 52), pp. 177-198.

Tapia, $\mathrm{M}$.

2017 "Las fronteras, la movilidad y lo transfronterizo: Reflexiones para un debate”. Estudios Fronterizos, 18 (37), 61-80.

Tarrius, A.

2000 "Leer, describir, interpretar las circulaciones migratorias: conveniencia de la noción de 'territorio circulatorio'. Los nuevos hábitos de la identidad”. Relaciones, 21 ( $\mathrm{N}^{\circ} 83$ ), pp. 37-66.

Tarrius, A.

2001 Au-delà des États-nations : des sociétés de migrants. In: Revue européenne des migrations internationales, Débats contemporains, sous la direction de Jacqueline Costa-Lascoux et Marie-Antoinette Hily. 17( ${ }^{\circ}$ 2), pp. 37-61.

Timur, S.

2000 Cambios de tendencia y problemas fundamentales de la migración internacional: una perspectiva general de los programas de la UNESCO. Revista internacional de ciencias sociales. UNESCO, $\mathrm{N}^{\circ} 165$.

Zusman, $\mathrm{P}$

2013 "La geografía histórica, la imaginación y los imaginarios geográficos”. Revista de Geografía Norte Grande ( $\left.\mathrm{N}^{\circ} 54\right)$, pp. 51-66. 


\section{Notas}

1 Resultado proyecto: "Política criminal y migración en el norte de Chile" financiado por el programa: PAI-MEC de Conicyt, folio 80160013 y de la investigación doctoral de Romina Ramos (2017) titulada "La frontera como dispositivo de control: análisis de la construcción material y simbólica de las prácticas transfronterizas en TarapacáChile”. Universidad del País Vasco. España.

2 Paasi (2009) ha puesto en cuestión lo que sería el análisis de los "territorios limitados" cuya expresión debe entenderse como un "tipo de espacialidad de poder". Daniel Neep (2016) también describe el "fetichismo espacial del territorio" refiriéndose a que es interpretado como un espacio abstracto del Estado moderno, inerte y homogéneo (en su interior) y fijo en los límites exteriores que caracterizan al Estado moderno capitalista, donde la homogeneidad es útil para la circulación de mercancías. También Benedetti (2011b) critica esta comprensión del territorio y señala que las ciencias sociales se han involucrado en una especie de "fetichismo territorial" al suponer que el territorio es producido exclusivamente por el Estado, desconociendo otras no territorialidades que se manifiestan, incluso, a partir del ejercicio del propio Estado.

3 En adelante, el concepto región lo utilizaremos como sinónimo de la organización social territorial de nuestro espacio de interés. Si bien reconocemos la distinción entre lo físico-espacial y lo social-espacial -en cuanto al tratamiento del concepto región- por tratarse de una aproximación desde una perspectiva fenomenológica, adherimos a la noción social espacial propuesta por Henri Lefebvre (1974).

$4 \quad$ Esta complejidad trae consigo una dialéctica conflictiva y continua de reproducción social, que determina las disposiciones del habitus en los mecanismos de posesión y posición en el espacio que produce territorio (Bourdieu, 1999).

5 La idea de espacio fronterizo más flexible, que incorporamos a nuestro estudio, incluye al límite establecido por dos entidades que, en simultáneo, ejercen su territorialidad (Benedetti, 2014).

6 Entendemos las regiones fronterizas, bajo el marco que se intenta proponer para efectos de este estudio, como aquellas que están determinadas por los procesos sociales que se establecen en estos espacios donde los límites internacionales (línea fronteriza) se convierten en factores que dinamizan las relaciones de intercambio y comercio transfronterizo, lejos del rol formal que les asignan los tratados y las leyes.

$7 \quad$ El acceso mediante otro mecanismo de contactación era casi imposible, ya que el perfil de los entrevistados que resultaban de nuestro interés contaba con varias características que hacían difícil su contactación por una vía "formal" o por medio de la búsqueda de perfiles sociodemográficos específicos. Principalmente, son personas que mantienen una situación de irregularidad en el estatuto de su documentación, por lo que esta forma de contactación inspiraba para los entrevistados cruzadores fronterizos un cierto grado de confianza para acceder a la entrevista.
8 La concepción de soberanía del territorio, que ha predominado en la teoría política moderna, se basa en la idea de la autoridad política exclusiva ejercida por un Estado en un territorio determinado (Agnew, 2005). Aunque es una concepción ortodoxa del término, nos sirve para contextualizar su inicio. Las principales formas en las que se ejerce actualmente la soberanía: (1) su construcción social; (2) su asociación con la subordinación jerárquica y (3) su despliegue en formas territoriales y no territoriales (Agnew,2006: 88). La soberanía necesita agentes dependientes para poder ejercerla. Lo evidente, sin embargo, es que la trillada aseveración del "fin de la geografía", por un lado, y nada ha cambiado en el mundo, el territorio sigue siendo la base de la política mundial, por el otro, se mantienen en la necesidad de confrontación y reemplazo.

9 Las principales ciencias sociales en las universidades occidentales contemporáneas fueron fundadas para prestar servicios intelectuales a los Estados modernos con el fin de crear riqueza, controlar la sociedad y administrar el Estado, respectivamente (Benedetti, 2011). Por consiguiente, no es extraño que las ciencias sociales encuentren, en cierto modo, que es problemático moverse fuera de un mundo incuestionablemente dividido en unidades concretas de territorio soberano. Un ejemplo de aquello ha sido el rol de la geografía política que al estar supuestamente relacionada con la espacialidad del poder, ha permanecido durante bastante tiempo vinculada con una imaginación geopolítica basada en concebir el poder coercitivo y el Estado territorial como indicadores de la modernidad: "Corregir esta concepción es uno de los retos fundamentales a los que se enfrenta la geografía política de principios del siglo XXI" (Agnew, 2005: 78).

10 Ana, boliviana, cruce por la frontera Colchane, visa de turismo (entrevista 15 de mayo de 2015).

11 Rosario, boliviana, cruce por la frontera de Colchane, visa de turismo (entrevista el 5 de mayo de 2015).

12 La migración temporal que vemos en los relatos anteriores, en sus distintas modalidades, así como "trabajadores invitados", "trabajadores temporeros" y "trabajadores contratados", se generalizó en las sociedades avanzadas como resultado de su supuesta flexibilidad y eficiencia a la hora de hacer frente a lo que se percibía como una escasez temporal de mano de obra (Timur, 2000).

13 Encargado de funciones administrativas de la gobernación provincial del Tamarugal en complejo fronterizo Colchane (entrevista 13 de enero de 2015).

14 Soledad, bolviana, cruce por la frontera Colchane, visa de turismo (entrevista el 16 de abril de 2015).

15 Elena, colombiana, cruce por la frontera de Colchane, irregular en su estatus administrativo (entrevista el 15 de enero de 2015).

16 Encargado de Policía de Investigaciones en Complejo fronterizo Colchane (entrevista el 13 de enero de 2015).

17 María, boliviana, cruce por frontera Colchane, visa temporaria (entrevista el 17 de enero de 2015). 